\title{
Camrelizumab for the treatment of advanced cervical adenocarcinoma: a case report and literature review
}

\author{
Lei Zhang ${ }^{1}$, Yuqi Jiang ${ }^{1}$, Chaofan Xue ${ }^{2}$, Huiyong Chen ${ }^{3}$, Yongchun Zhang ${ }^{3}$ \\ ${ }^{1}$ Radiation Oncology, Qingdao University, Qingdao, China; ${ }^{2}$ Department of Oncology Hematology, Peoples Hospital of Qingdao West Coast New \\ Area, Qingdao, China; ${ }^{3}$ Department of Radiation Oncology, The Affiliated Hospital of Qingdao University, Qingdao, China \\ Correspondence to: Yongchun Zhang. Department of Radiation Oncology, The Affiliated Hospital of Qingdao University, 16 Jiangsu Road, Qingdao \\ 266003, China. Email: zyc18661805058@163.com.
}

\begin{abstract}
Cervical adenocarcinoma belongs to an invasive subtype of cervical carcinoma, presenting poorly prognostic status. Chemotherapy treatment for recurrent cervical carcinoma are thought to be limited and supposed to be noncurative. Because of the poor prognosis of patients with recurrent cervical carcinoma, however, the benefits of second-line chemotherapy have not yet reached a consensus. Immunotherapy is a split-new tactic of overwhelming carcinomas that relies on the instinct of the immune system to recognize and directly kill neoplasm cells. Here, we reported a 55-year-old female patient with clinical stage IVB cervical adenocarcinoma. The patient received four cycles of systematic therapy, with the regimen of docetaxel plus carboplatin in combined with bevacizumab anti-vascular therapy. The progressive disease (PD) was assessed by imaging evaluation and PD was confirmed once more after four cycles of chemotherapy of albumin paclitaxel plus cisplatin. The patient exhibited a good response during the twelve-cycle of immunotherapy of Camrelizumab, whereas PD was observed upon termination of her immunotherapy. This case with the treatment of PD-1 inhibitor Camrelizumab exhibits a good curative effect and tolerable adverse reactions. In addition, some clinical markers and biomarkers expression levels can be served as the predictors of the effect of anti-PD-1 immunotherapy.
\end{abstract}

Keywords: Advanced cervical adenocarcinoma; camrelizumab; immunotherapy; case report

Submitted Nov 22, 2021. Accepted for publication Jan 19, 2022.

doi: 10.21037/atm-22-67

View this article at: https://dx.doi.org/10.21037/atm-22-67

\section{Introduction}

Cervical cancer is the fourth most common malignancy in women, and its incidence ranks first among female reproductive system tumors, with 604,000 new cases and 342,000 deaths recorded worldwide in 2020. The incidence of cervical adenocarcinoma has been increasing in recent years, accounting for about $10-20 \%$ of new cases of cervical cancer (1). Most cervical adenocarcinomas originate in the cervical canal and are classified by International Endocervical Adenocarcinoma Criteria and Classification (IECC) as human papillomavirus (HPV)associated adenocarcinomas (HPVAs) and non-HPVassociated adenocarcinomas (NHPVAs). The HPVAs can be classified as common type, mucinous type, infiltrative laminar mucinous type, and so on, whereas NHPVAs can be classified as gastric type, clear cell type, mesonephric ductal type, and so forth (2). Cervical adenocarcinoma has the following particular behaviors compared to squamous carcinoma (2): (I) various pathological types, (II) insidious onset and difficult to diagnose at the early stage, (III) stronger invasiveness and endogenous growth, vascular invasion, and lymph node metastasis, (IV) less sensitive to radiotherapy. Therefore, it is generally accepted that the prognosis of cervical adenocarcinoma is less favorable than that of squamous carcinoma, and the prognosis of poorly differentiated cervical adenocarcinoma is less favorable than that of highly differentiated. At present, the treatment of cervical adenocarcinoma is no different from that of 
squamous carcinoma. Surgical treatment is available for stage I to stage IIA cervical cancer. For advanced stage cervical cancer (stage IIB and beyond), its treatment is mainly systemic treatment (platinum-based chemotherapy) \pm local management (radiotherapy of lesions and lymph nodes), but there are few options for patients whose disease progresses after first-line chemotherapy. Immunotherapy against PD-1/PD-L1 is the current hot spot in cervical cancer treatment and is expected to improve the treatment outcome of cervical adenocarcinoma. Camrelizumab, a humanized monoclonal antibody against PD-1, has been used in a variety of solid tumors such as nasopharyngeal carcinoma and hepatocellular carcinoma due to its favorable clinical activity and safety profile, but has fewer studies in cervical cancer. We report a patient with cervical adenocarcinoma with stage IVB, and the patient is deserving of being reported for the following reasons: (I) the patient was diagnosed with advanced ADC, a subtype of cervical cancer with a poor prognosis, (II) effective control of tumor with only camrelizumab immunotherapy after failure of both first- and second-line chemotherapy, and at least 10 months of PFS, (III) the patient has PD-L1-negative which is considered unfavorable for immunotherapy, but has MLH1 gene mutation, which could be a target for predicting immunotherapy efficacy. We present the following article in accordance with the CARE reporting checklist (available at https://atm.amegroups.com/article/ view/10.21037/atm-22-67/rc).

\section{Case presentation}

A 55-year-old female patient presented with "irregular vaginal fluid for more than 1 year" at the Affiliated Hospital of Qingdao University in May 2019. Gynecologic examination indicated cauliflower-like swelling of the cervix (approximately $4 \times 5 \mathrm{~cm}$ in diameter), thickening of the anterior fornix of the cervix, bilateral thickening of the primary sacral ligament, and invasion of the pelvic wall. The pathology of cervical biopsy showed poorly differentiated adenocarcinoma, and the results of immunohistochemical (IHC) examination showed that the lesion was cytokeratin (CK) 8/18 positive $(+)$, carcinoembryonic antigen (CEA) $(+)$, CD56 weak (+), p63 negative (-), p40 (-), thyroid transcription factor (TTF)-1 (-), and CK5/6 negative (-). Cervical fluid-based cytology revealed negativity for human papillomavirus. The patient had no family history of cervical cancer.

The results of whole-body positron emission tomography-computed tomography (PET-CT) revealed soft tissue density shadow of the cervix, invading the body and fundus of the uterus, approximately $78 \mathrm{~mm}$ in diameter, and standardized uptake value $\left(\mathrm{SUV}_{\max }\right)$ was 23.3 ; multiple soft tissue density shadow of the vaginal wall, the largest of which is approximately $40 \mathrm{~mm}$ in diameter, and $\mathrm{SUV}_{\text {max }}$ was 8.1; multiple soft tissue density shadow of both lungs, the largest of which was approximately $16 \mathrm{~mm}$ in diameter, and $\mathrm{SUV}_{\text {max }}$ was 12.7; and multiple enlarged lymph nodes in the bilateral iliac vascular area and adjacent to the abdominal aorta $\left(\mathrm{SUV}_{\max }\right.$ was 4.5$)$, bilateral hilum and mediastinum $\left(\mathrm{SUV}_{\text {max }}\right.$ was 18.5), as shown in Figure 1.

The results of next generation sequencing (NGS) of blood and tumor showed microsatellite stability (MSS), and low (2.21 mutations/Mb) tumor mutation burden (TMB). The IHC indicated low expression (1\%) of programmed death receptor ligand-1 (PD-L1) and positive expression of immune-related genes $M L H 1$, as shown in Table 1.

Based on the International Federation of Gynecology and Obstetrics (FIGO) cervical cancer staging (2018 edition), the clinical diagnosis of this case was poorly differentiated cervical adenocarcinoma of stage IVB with metastasis to non-regional lymph nodes and lung.

The patient started first-line treatment with "paclitaxel + carboplatin (TC) combined with bevacizumab" in May 2019 at the following dosage: docetaxel $100 \mathrm{mg}$ day $1+$ carboplatin $400 \mathrm{mg}$ day 1 + bevacizumab $500 \mathrm{mg}$ day 1 , intravenous drip, repeated every 3 weeks. After 2 cycles of chemotherapy, the patient underwent CT examination (9 July 2019), and the efficacy was evaluated as partial remission (PR) for both primary and metastatic foci. This regimen of chemotherapy was continued. After 4 cycles of chemotherapy, pelvic CT (26 August 2019), showed an enlarged cervical tumor with invasion of the uterine body, vagina, and left ureter, and a progressive metastasis in both lungs compared to after cycle 2 (9 July 2019), which was evaluated as tumor progressive disease (PD). The 5th cycle of chemotherapy was switched to "albumin paclitaxel + cisplatin (TP)" regimen (bevacizumab was discontinued due to the patient's financial constraints) at the following dose: albumin paclitaxel $300 \mathrm{mg}$ day $1+$ cisplatin $50 \mathrm{mg}$ day 1 and 2 , intravenous drip, repeated every 3 weeks. Efficacy was evaluated as PR after 2 cycles of second-line chemotherapy (18 October 2019) and re-evaluated as PD after 4 cycles (17 December 2019), compared to the CT on 18 October 2019 , for both primary and pulmonary metastatic foci. Considering resistance to the second-line regimen, after adequate communication with the patient, she agreed 

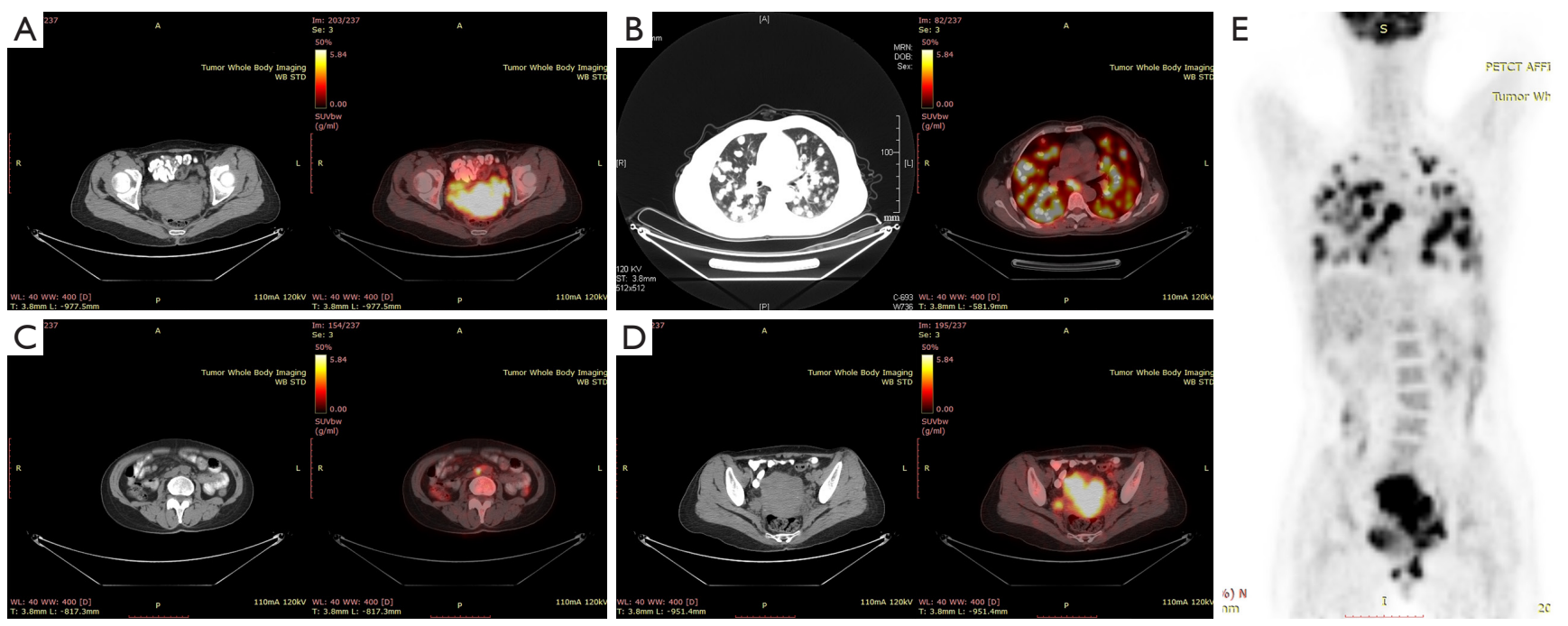

Figure 1 PET-CT result of the patient. Hypermetabolic foci in the cervix, vagina, iliac vessels, para-aortic lymph nodes, and lung. (A) Primary foci in the cervix; (B) multiple metastases in the lung; (C) para-aortic hypermetabolic lymph nodes; (D) para-iliac vessels hypermetabolic lymph nodes and uterine cavity invasion; (E) the patient's whole body hypermetabolic distribution. PET-CT, positron emission tomography-computed tomography.

Table 1 Result of gene detection in blood and tissue samples of the patient

\begin{tabular}{lc}
\hline Characteristics & Results \\
\hline Blood sample & MLH1 p.R470K \\
Associated mutation genes & - \\
Positive & FGFR2 p.T764fs, AKT1 p.E17K, BCOR p.P1660L and TP53 p.R273H \\
Negative & Low expression, 1\% \\
Unknown significance & MSS, MSI score: 0.0156 \\
Tissue sample & Low (2.21 mutations/Mb) \\
PD-L1 expression & \\
MSI & \\
TMB & FGFR2 p.T764fs, AKT1 p.E17K, BCOR p.P1660L and TP53 p.R273H \\
Pssociated mutation genes
\end{tabular}

PD-L1, programmed death receptor ligand-1; MSS, microsatellite stability; MSI, microsatellite instability; TMB, tumor mutation burden.

to a trial of the PD-1 inhibitor camrelizumab. After 2 cycles (25 March 2020), both primary cervical focus and metastatic foci were evaluated as PR by enhanced CT; after 5 cycles (26 May 2020), the primary focus was evaluated as stable disease (SD) and pulmonary foci were evaluated as complete remission (CR); after 8 cycles (14 August 2020) the primary focus was evaluated as SD and pulmonary foci evaluated as CR. At that time, the patient was advised to receive pelvic radiotherapy, but she refused and continued immunotherapy. The last evaluation of the primary cervical 

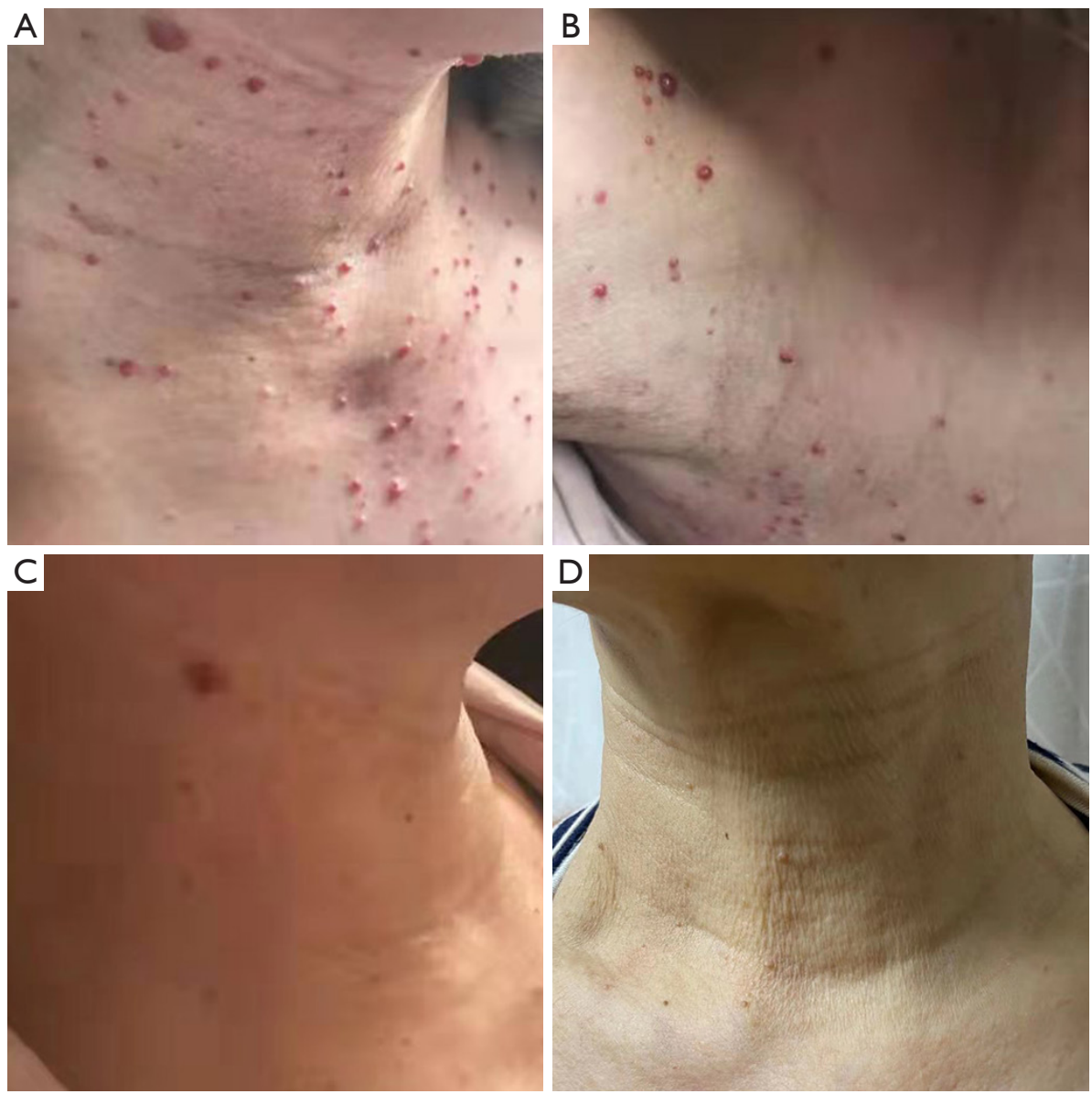

Figure 2 Condition and changes of RCCEP in the neck of the patient. RCCEP in the neck after 2 cycles of camrelizumab treatment. (A) 3 days after the occurrence of RCCEP; (B) 1 month after the occurrence of RCCEP; (C) 3 months after the occurrence of RCCEP; (D) 4 months after the occurrence of RCCEP. RCCEP, reactive cutaneous capillary hyperplasia.

focus was on 12 October 2020, with a result of SD, and the evaluation of the pulmonary foci was on 14 December 2020, with a result of CR.

At this time of treatment, the patient, and her family were aware of the illness and actively cooperated with the doctors. The patient had no significant discomfort, the adverse effects did not affect her daily life, and the treatment effect was considered to exceed expectation.

The patient stopped immunotherapy of her own accord in November 2020, after a total of 12 cycles of immunotherapy. The patient's CT and magnetic resonance (MR) results were reviewed on 20 January 2021: the primary focus was PD with enlarged lymph nodes in the retroperitoneum, and the pulmonary metastases remained CR, compared to the MR on 12 October 2020, and CT on 14 December 2020. At that time, the patient agreed to receive pelvic radiotherapy and continue immunotherapy.

The main immune-related adverse reaction during immunotherapy was reactive cutaneous capillary hyperplasia (RCCEP), which occurred after 2 cycles of immunotherapy, mainly in her neck and chest, in "red nevus type" and "pearl type", with an evaluation of grade 1 (diameter $<1 \mathrm{~cm}$ and no infection), no special treatment was performed because there was no rupture or bleeding, and it gradually subsided after 5 cycles, as shown in Figure 2.

The concentration of CA125 in venous blood during the patient's treatment appeared to change accordingly during the treatment, as shown in Figure 3. The images of the patient's successive evaluations during the treatment are shown in Figures 4-6. The patient's treatment timeline is shown in Figure 7.

All procedures performed in this patient were in 


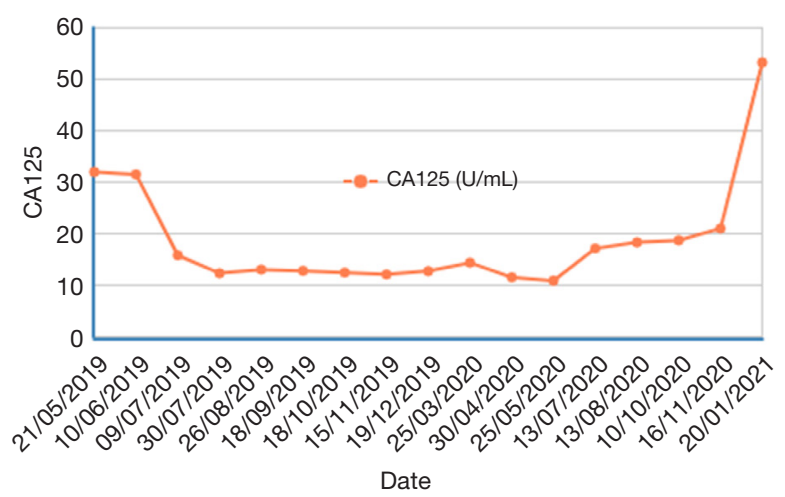

Figure 3 Changes in venous blood CA125 during the patient's treatment. The CA125 showed a tendency of declining to rising (and significantly over 1 month after stopping immunotherapy) during the treatment.

accordance with the ethical standards of the institutional and national research committees and with the Helsinki Declaration (as revised in 2013). Written informed consent was obtained from this patient for publication of this case report and accompanying images. A copy of the written consent is available for review by the editorial office of this journal.

\section{Discussion}

Since immune escape by tumor cells through high expression of PD-L1, immunotherapy represented by PD-1 inhibitors has shown promising efficacy in cervical cancer. The KEYNOTE-028 study by Piha-Paul et al. (3) showed a median survival of 9 months in patients with advanced cervical cancer treated with the PD-1 inhibitor pembrolizumab, and KEYNOTE-158 showed an objective response rate (ORR) of $14.3 \%$ with pembrolizumab in PDL1-positive cervical cancer patients, while no response was observed in the PD-L1-negative patients. Based on these studies, the U.S. Food and Drug Administration (FDA) approved pembrolizumab for the treatment of advanced and recurrent cervical cancer [indication for PD-L1 positive or microsatellite highly unstable (MSI-H)]. Fewer clinical studies have been conducted with camrelizumab in cervical cancer. However, all have achieved good efficacy: Lan et al. (4) applied camrelizumab in combination with apatinib for advanced cervical cancer and achieved an objective remission rate (ORR) of $55.6 \%$, but these drugs may have overlapping toxicity: $93.3 \%$ of patients had dose reduction or suspension of chemotherapy due to adverse effects such as apatinib-related fatigue, stomatitis, and myalgia. Zhang et al. (5) applied camrelizumab for advanced uterine malignancies (24 patients with cervical cancer and 7 patients with endometrial cancer), 22 patients $(70.97 \%)$ did not show tumor progression. Song (6) reported progressionfree survival of 6 and 10 months in 2 patients with liver metastases from cervical cancer treated with camrelizumab, respectively. In addition, Boussios et al. (7) reported that bevacizumab could enhance the anti-tumor effect of PD-1 inhibitors through neovascularization. However, Friedman et al. (8) did not achieve the desired effect in the study of PD-L1 inhibitor atezolizumab combined with bevacizumab in the treatment of cervical cancer.

A study of melanoma by Xiao et al. (9) showed that effective anti-PD-1/PD-L1 therapy was strongly associated with high PD-L1 expression, while Borghaei et al. (10) reported that PD-L1-negative patients also had a good prognosis.

The expression rate of PD-L1 in cervical cancer ranged from $53 \%$ to $88 \%$ (11), suggesting that immunotherapy may benefit most cervical cancer patients. However, conversely, the high expression rate of PD-L1 also suggests that using it as a marker to predict clinical benefits may be imprecise. Although all PD-L1-negative cervical cancer patients in the KEYNOTE-158 study did not respond to PD-1 inhibitors, the sample was small and needed to be validated in a more extensive scale. In this case, although the patient had low PD-L1 expression, she received a good clinical efficacy, so the impact of PD-L1 expression on the clinical efficacy of cervical adenocarcinoma is still worth further exploration.

The TP53 gene is a tumor suppressor, about $20 \%$ of patients harbor TP53 mutation (12), and its mutation rate is higher in patients with cervical adenocarcinoma than squamous. Piha-Paul and Biton et al. $(3,13)$ reported that mutations of this gene in lung adenocarcinoma were strongly associated with good efficacy of PD-1 inhibitors, while the effects were opposite in ovarian, esophageal, and colon cancers. No clinical study has confirmed the effect in cervical cancer, but basic research by Tornesello et al. (14) showed that TP53 plays an essential role in cervical adenocarcinoma, and high expression of this gene is also considered a predictor of poor prognosis in cervical cancer (15).

Mutations in the MLH1 gene, which plays a role in DNA mismatch repair, result in mismatch repair deficiency (dMMR) and MSI-H. Mandal et al. (16) used tumor-bearing mice to show that $\mathrm{dMMR} / \mathrm{MSI}-\mathrm{H}$ is a valuable marker for PD-1 inhibitors in various solid tumors, including cervical cancer, possibly because tumor cells with MSI-H 

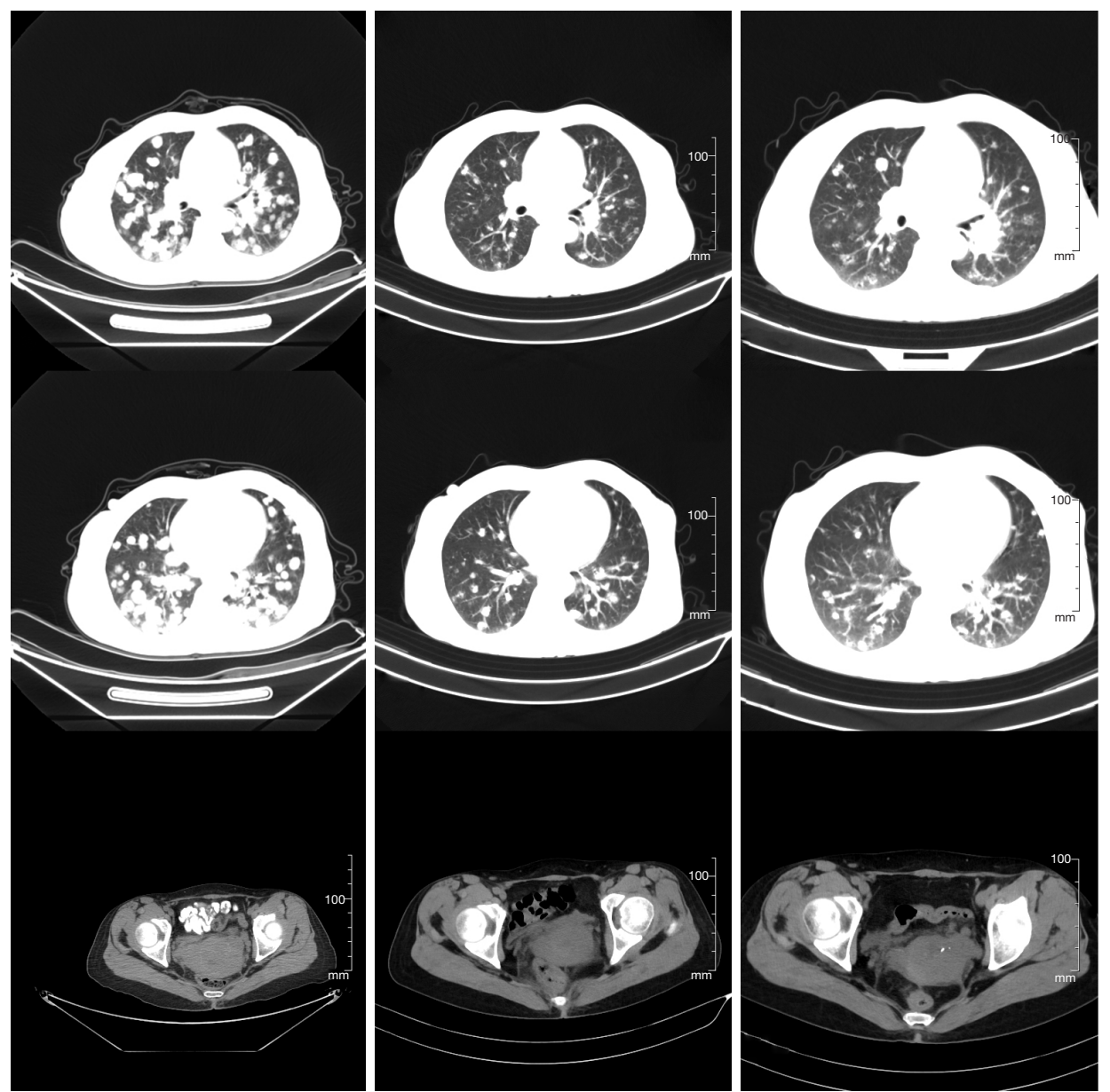

Figure 4 Comparison of primary cervical and pulmonary metastatic foci during the first-line treatment of the patient. PET-CT, positron emission tomography-computed tomography.

produce more cancer-related antigens and are susceptible to the immune system (17). Le et al. (18) showed a $53 \%$ response rate to $\mathrm{PD}-1$ inhibitor therapy in patients with $\mathrm{dMMR} / \mathrm{MSI}-\mathrm{H}$ in various solid tumors, including colon and pancreatic cancers. No clinical studies of MLH1 have reported its effect in cervical cancer.

There are also other genes predictive of immune efficacy: Kato et al. (19) concluded that patients with solid tumors with mutations of TERT, PTEN, NF1, and NOTCH1 had better results with anti-PD-1/PD-L1 immunotherapy and those with mutations of $M D M 2, M D M 4$, and DNMT3A had more unsatisfactory efficacy. Pore et al. (20) found that SKT11 mutation was unfavorable for anti-PD-1/PDL1 immunotherapy but could be reversed by STAT3targeted therapy. Lau et al. (21) concluded that patients with POLE mutation were susceptible to anti-PD-1/PD-L1 immunotherapy.

The use of each biomarker alone to predict the efficacy of PD-1 inhibitors is currently controversial, and there is no consensus on the predictivity of multiple markers in combination (22).

Our decision to treat this patient with camrelizumab was based on a combination of these factors: (I) she had advanced cervical adenocarcinoma and was resistant to both first- and second-line therapies, leaving few treatment options; (II) clinical studies have shown that PD-L1negative patients may also benefit from anti-PD-1/ PD-L1 immunotherapy; (III) the patient had an MLH1 gene mutation, and fundamental studies support the use of this mutation in patients with anti-PD-1/PD-L1 


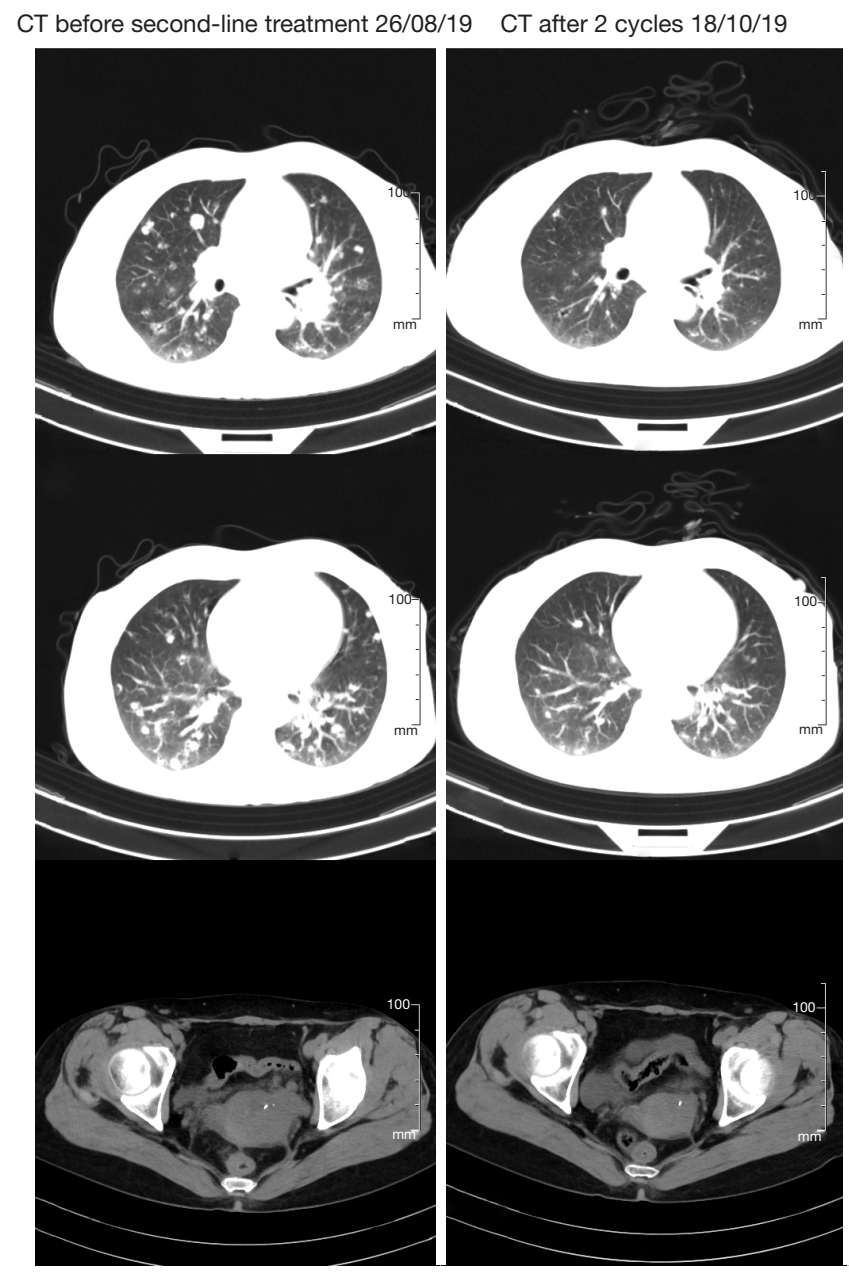

CT after 4 cycles 17/12/19

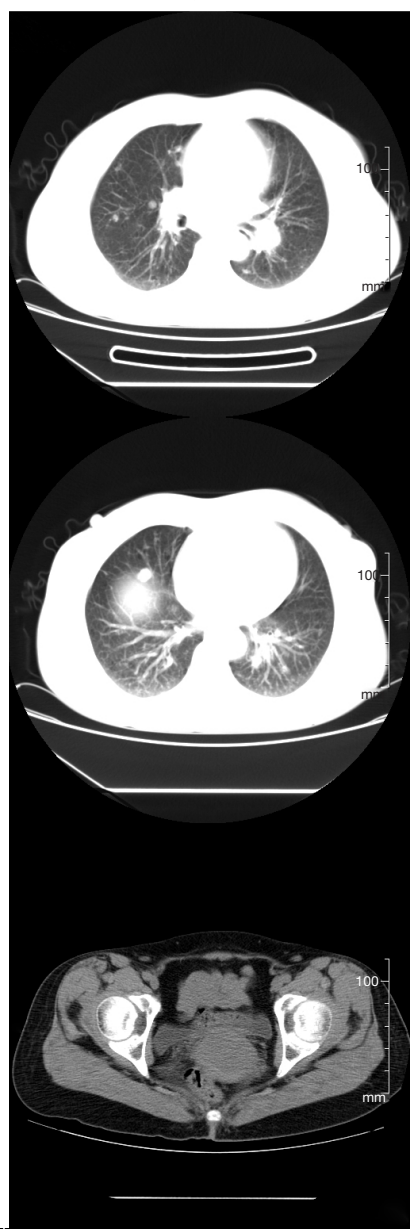

Figure 5 Comparison of primary cervical and pulmonary metastatic foci during the second-line treatment of the patient. CT, computed tomography.

immunotherapy.

Camrelizumab triggers a high proportion of AEs (97\%, of which $24 \% \geq$ grade 3 ), among which RCCEP, anemia, fever, fatigue, hypothyroidism, cough, and proteinuria are the most common complications. Most of these AEs are reversible, and prevention and early recognition are the most important factors in treating these side effects. The RCCEP is a typical adverse reaction of camrelizumab (23), which occurs in around 1 month after drug administration, is self-limiting (healing after 3-6 months without scarring), and occurs only on the skin, rather than in visceral mucosa. Morphologically, RCCEP is mainly classified as red nevus, pearl, mulberry, patchy, and tumor-like type. Mechanism of RCCEP occurrence may be that camrelizumab reactivates the immune response by blocking the immunosuppressive pathway, and the overactivation of this process stimulates CD4+ and Th2 cells to secrete interleukin (IL)-4 and macrophage colony-stimulating factor (M-CSF), which promote the differentiation and expansion of macrophages in the skin toward the M2 type and release vascular endothelial growth factor (VEGF)-A, ultimately causing abnormal capillary proliferation. The treatment of RCCEP is mainly symptomatic, and the traditional Chinese medicine (TCM) formula Yunnan Baiyao or antibiotics can be used externally in the event of ulceration. A study by Wang et al. (24) in patients with hepatocellular carcinoma showed that those who developed RCCEP had better clinical efficacy and survival benefit, and the development of RCCEP in cervical cancer may also serve as a clinical marker for predicting the efficacy of camrelizumab. 


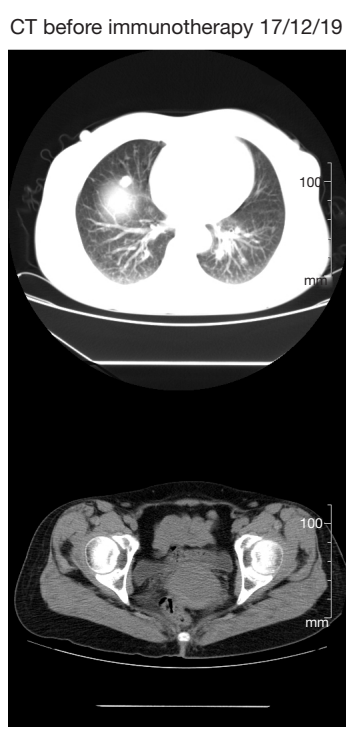

CT after 2 cycles 25/03/20

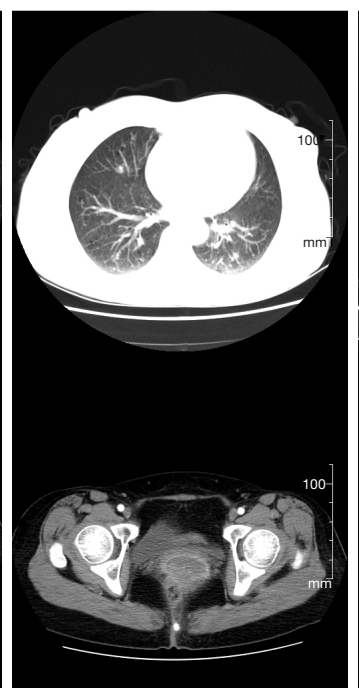

CT/MR after 5 cycles 26/05/20 CT/MR before PD 14/12/20 and 12/10/20

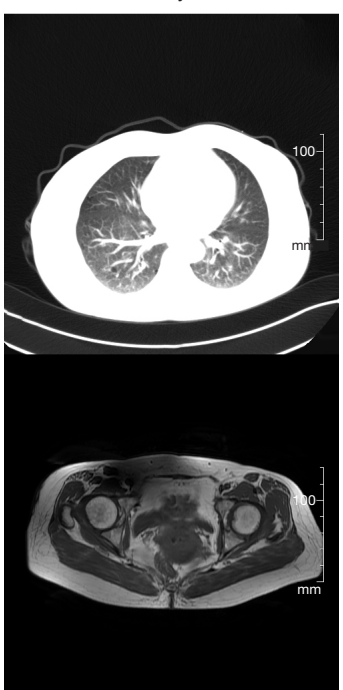

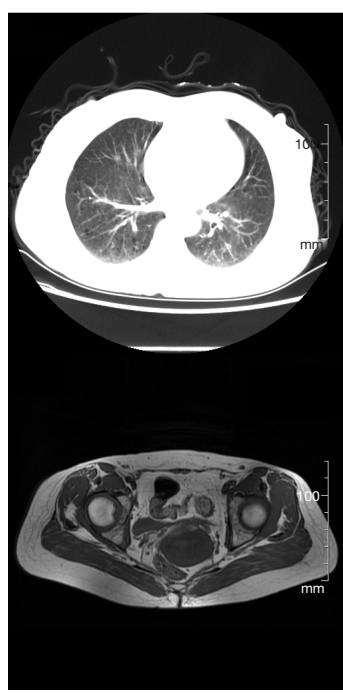

CT/MR after PD 22/01/21

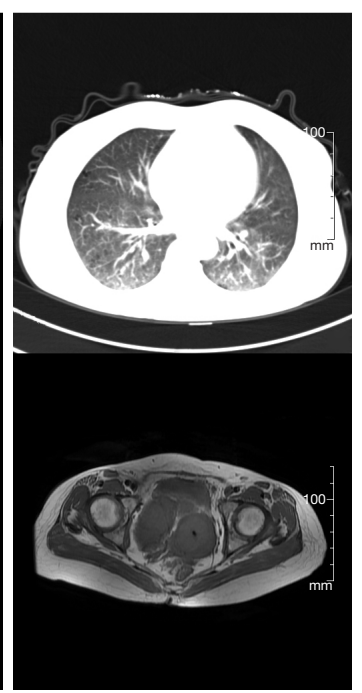

Figure 6 Comparison of primary cervical and pulmonary metastatic foci during the immunotherapy of the patient. CT, computed tomography; MR, magnetic resonance; $\mathrm{PD}$, progressive disease.

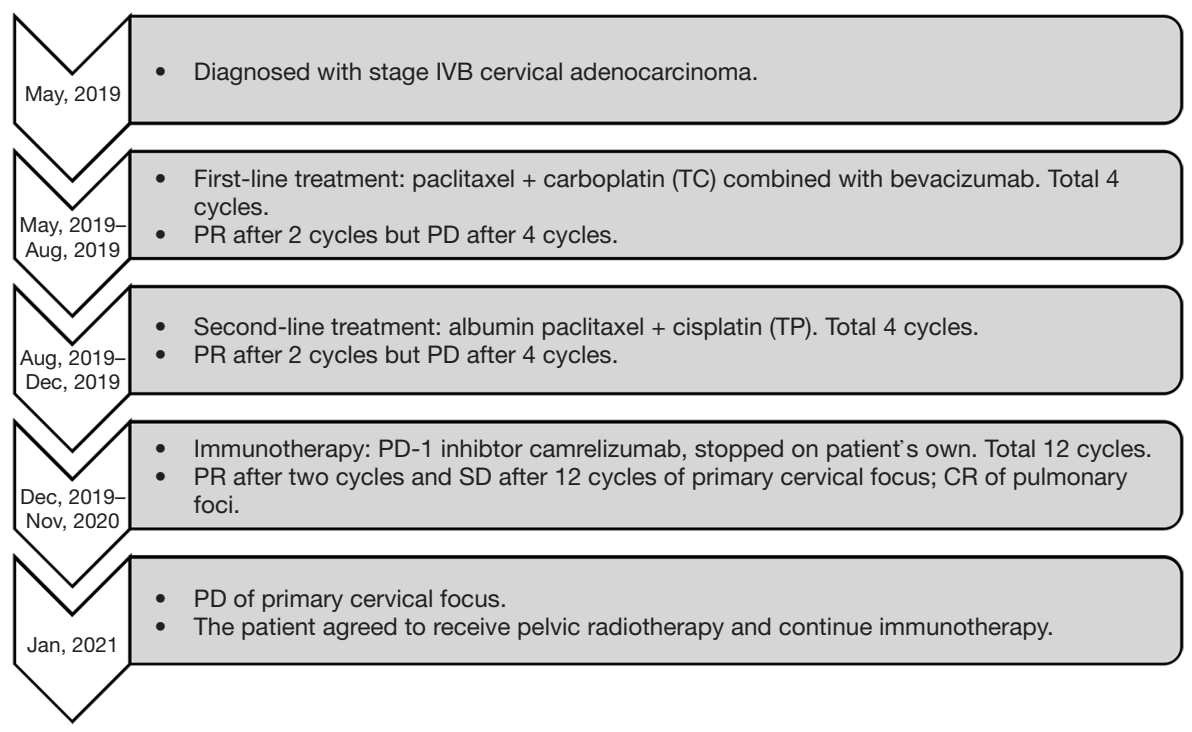

Figure 7 Timeline of the patient's treatment. PD, progressive disease; PR, partial response; SD, stable disease; CR, complete response.

This case shows that patients with progressive advanced cervical adenocarcinoma are well treated with the PD-1 inhibitor camrelizumab, with a long duration of disease control and mild adverse effects. Unfortunately, this patient's tumor progressed again after self-discontinuation of immunotherapy, mainly due to progression of the primary focus. Based on this case, we suggest the following for the treatment of advanced cervical adenocarcinoma:
(I) early detection of immune checkpoints such as PDL1 to clarify the possibility of immunotherapy; (II) even if patients have low PD-L1 expression, PD-1/PD-L1 inhibitor such as camrelizumab can be tried; (III) for patients with effective immunotherapy, maintenance therapy and supplementation with local radiotherapy may allow patients to obtain longer PFS.

We draw the following conclusions from the case: (I) 
immunotherapy with camrelizumab after resistance to first-line chemotherapy is effective and can be used as a reference for subsequent line treatment in advanced cervical adenocarcinoma; (II) detection of PD-L1, TMB, MSI, and other immune-related genes is recommended to predict the effect of immunotherapy; (III) MLH1 mutation or RECCP appearing in the process of immunotherapy may be the positive markers of the curative effect of camrelizumab. In addition, the progression-free survival time of this patient may increase with the addition of pelvic radiotherapy when immunotherapy achieves better control.

\section{Acknowledgments}

Funding: None.

\section{Footnote}

Reporting Checklist: The authors have completed the CARE reporting checklist. Available at https://atm.amegroups. com/article/view/10.21037/atm-22-67/rc

Conflicts of Interest: All authors have completed the ICMJE uniform disclosure form (available at https://atm. amegroups.com/article/view/10.21037/atm-22-67/coif). The authors have no conflicts of interest to declare.

Ethical Statement: The authors are accountable for all aspects of the work in ensuring that questions related to the accuracy or integrity of any part of the work are appropriately investigated and resolved. All procedures performed in this patient were in accordance with the ethical standards of the institutional and national research committees and with the Helsinki Declaration (as revised in 2013). Written informed consent was obtained from the patient for publication of this case report and accompanying images. A copy of the written consent is available for review by the editorial office of this journal. Due to the retrospective and non-interventional nature of the study, permission by the local ethics committee was not required.

Open Access Statement: This is an Open Access article distributed in accordance with the Creative Commons Attribution-NonCommercial-NoDerivs 4.0 International License (CC BY-NC-ND 4.0), which permits the noncommercial replication and distribution of the article with the strict proviso that no changes or edits are made and the original work is properly cited (including links to both the formal publication through the relevant DOI and the license). See: https://creativecommons.org/licenses/by-nc-nd/4.0/.

\section{References}

1. Sung H, Ferlay J, Siegel RL, et al. Global Cancer Statistics 2020: GLOBOCAN Estimates of Incidence and Mortality Worldwide for 36 Cancers in 185 Countries. CA Cancer J Clin 2021;71:209-49.

2. Hodgson A, Olkhov-Mitsel E, Howitt BE, et al. International Endocervical Adenocarcinoma Criteria and Classification (IECC): correlation with adverse clinicopathological features and patient outcome. J Clin Pathol 2019;72:347-53.

3. Piha-Paul SA, Oh DY, Ueno M, et al. Efficacy and safety of pembrolizumab for the treatment of advanced biliary cancer: Results from the KEYNOTE-158 and KEYNOTE-028 studies. Int J Cancer 2020;147:2190-8.

4. Lan C, Shen J, Wang Y, et al. Camrelizumab Plus Apatinib in Patients With Advanced Cervical Cancer (CLAP): A Multicenter, Open-Label, Single-Arm, Phase II Trial. J Clin Oncol 2020;38:4095-106.

5. Zhang YX, Lou HM, Lv XJ, et al. Efficacy and safety of camrelizumab in patients with recurrent or advanced cervical cancer and endometrial cancer. J Chinese Journal of Critical Care Medicine (Electronic Edition) 2021;14:127-32.

6. Song G. Report of 2 cases of cervical cancer with liver metastasis treated with camrelizumab. J Anhui Medical Journal 2021;42:350-1.

7. Boussios S, Seraj E, Zarkavelis G, et al. Management of patients with recurrent/advanced cervical cancer beyond first line platinum regimens: Where do we stand? A literature review. Crit Rev Oncol Hematol 2016;108:164-74.

8. Friedman CF, Snyder Charen A, Zhou Q, et al. Phase II study of atezolizumab in combination with bevacizumab in patients with advanced cervical cancer. J Immunother Cancer 2020;8:e01126.

9. Xiao W, Du N, Huang T, et al. TP53 Mutation as Potential Negative Predictor for Response of AntiCTLA-4 Therapy in Metastatic Melanoma. EBioMedicine 2018;32:119-24.

10. Borghaei H, Gettinger S, Vokes EE, et al. Five-Year Outcomes From the Randomized, Phase III Trials CheckMate 017 and 057: Nivolumab Versus Docetaxel in Previously Treated Non-Small-Cell Lung Cancer. J Clin Oncol 2021;39:723-33.

11. Zong LJ, Xiang Y. Research progress and future of 
immunotherapy for advanced and recurrent cervical cancer. Chinese Journal of Practical Gynecology and Obstetrics 2018;34:1211-6.

12. Zhao ZQ, Liang XM, Zhang FY, et al. Analysis of p53 mutation, co-expression network and clinical outcome in cervical cancer tumor tissues based on bioinformatics technology. Chinese Medicinal Biotechnology 2019;14:27-32.

13. Biton J, Mansuet-Lupo A, Pécuchet N, et al. TP53, STK11, and EGFR Mutations Predict Tumor Immune Profile and the Response to Anti-PD-1 in Lung Adenocarcinoma. Clin Cancer Res 2018;24:5710-23.

14. Tornesello ML, Buonaguro L, Buonaguro FM. Mutations of the TP53 gene in adenocarcinoma and squamous cell carcinoma of the cervix: a systematic review. Gynecol Oncol 2013;128:442-8.

15. Gaber G, El Achy S, Khedr GA, et al. Impact of p53, HIF1a, Ki-67, CA-9, and GLUT1 Expression on Treatment Outcomes in Locally Advanced Cervical Cancer Patients Treated With Definitive Chemoradiation Therapy. Am J Clin Oncol 2021;44:58-67.

16. Mandal R, Samstein RM, Lee KW, et al. Genetic diversity of tumors with mismatch repair deficiency influences antiPD-1 immunotherapy response. Science 2019;364:485-91.

17. Overman MJ, Lonardi S, Wong KYM, et al. Durable Clinical Benefit With Nivolumab Plus Ipilimumab in DNA Mismatch Repair-Deficient/Microsatellite Instability-High Metastatic Colorectal Cancer. J Clin Oncol 2018;36:773-9.

18. Le DT, Durham JN, Smith KN, et al. Mismatch repair deficiency predicts response of solid tumors to PD-1

Cite this article as: Zhang L, Jiang Y, Xue C, Chen H, Zhang Y. Camrelizumab for the treatment of advanced cervical adenocarcinoma: a case report and literature review. Ann Transl Med 2022;10(4):239. doi: 10.21037/atm-22-67 blockade. Science 2017;357:409-13.

19. Kato S, Goodman A, Walavalkar V, et al. Hyperprogressors after Immunotherapy: Analysis of Genomic Alterations Associated with Accelerated Growth Rate. Clin Cancer Res 2017;23:4242-50.

20. Pore N, Wu S, Standifer N, et al. Resistance to durvalumab and durvalumab plus tremelimumab is associated with functional STK11 mutations in nonsmall-cell lung cancer patients and is reversed by STAT3 knockdown. Cancer Discov 2021;11:2828-45.

21. Lau D, Kalaitzaki E, Church DN, et al. Rationale and design of the POLEM trial: avelumab plus fluoropyrimidine-based chemotherapy as adjuvant treatment for stage III mismatch repair deficient or POLE exonuclease domain mutant colon cancer: a phase III randomised study. ESMO Open 2020;5:e000638.

22. Cristescu R, Mogg R, Ayers M, et al. Pan-tumor genomic biomarkers for PD-1 checkpoint blockade-based immunotherapy. Science 2018;362:eaar3593.

23. Chen X, Ma L, Wang X, et al. Reactive capillary hemangiomas: a novel dermatologic toxicity following anti-PD-1 treatment with SHR-1210. Cancer Biol Med 2019;16:173-81.

24. Wang F, Qin S, Sun X, et al. Reactive cutaneous capillary endothelial proliferation in advanced hepatocellular carcinoma patients treated with camrelizumab: data derived from a multicenter phase 2 trial. J Hematol Oncol 2020;13:47.

(English Language Editor: J. Jones) 